\title{
Benchmark correlation energies for small molecules
}

\author{
DARRAGH P. O'NEILL and PETER M. W. GILL* \\ School of Chemistry, University of Nottingham, Nottingham NG7 2RD, UK
}

(Received 29 June 2004; in final form 5 August 2004)

\begin{abstract}
We present estimates of the exact correlation energies for 56 small molecules whose experimental atomization energies are known accurately. These should prove useful in the assessment and parameterization of new quantum chemical methods.
\end{abstract}

\section{Introduction}

The prediction of thermochemical quantities to high accuracy $\left(\sim 1 \mathrm{kcal} \mathrm{mol}^{-1}\right)$ is an important and challenging goal in quantum chemistry and a purely $a b$ initio approach requires the use of highly accurate and computationally expensive treatments of electron correlation and relativistic effects. Many of the popular, more computationally accessible, but more empirical quantum chemical models, such as B3LYP [1] or the Gaussian- $n$ methods [2-5], are parameterized to reproduce such thermochemical data and while this is clearly a desirable quality in any method, an even more satisfying target is to reproduce accurately atomic and molecular total energies: this too would lead to accurate thermochemistry, but without depending upon unphysical error cancellation. Clearly, to parameterize or to assess the performance of a method requires an accurate data set. Although there are papers [6-11] which contain estimates of the total energy of one or several small molecules, we are not aware of a large systematic set of such data in the chemical physics literature. In this article we use a combination of accurately determined experimental and theoretical quantities to do so for 56 small molecules, viz. the 55 neutral molecules in the G1 set [2, 3] and $\mathrm{H}_{2}$. In particular, we list the non-relativistic (NR) total and electronic energies, the restricted and unrestricted Hartree-Fock (HF) energies and the corresponding correlation energies. We aim to determine these quantities to within a millihartree $\left(\mathrm{m} E_{\mathrm{h}}\right)$.

*Corresponding author. e-mail: p.gill@anu.edu.au. Present address: Research School of Chemistry, Australian National University, ACT 0200, Australia.

\section{Method}

The atomization energy, $\Sigma D_{0}$, of an $N$-atom molecule $\mathrm{M}$ is calculated using

$$
\Sigma D_{0}=\sum_{i}^{N} E_{\mathrm{e}}^{i}-E_{0}^{\mathrm{M}}+\Delta E_{\mathrm{Rel}},
$$

where $E_{\mathrm{e}}^{i}$ is the total NR electronic energy of the $i$ th atom in $\mathrm{M}, E_{0}^{\mathrm{M}}$ is the NR energy of the lowest vibronic state of the molecule, and $\Delta E_{\mathrm{Rel}}$ corrects the calculated atomization energy for the effects of relativity. $E_{0}^{\mathrm{M}}$ is given by

$$
E_{0}^{\mathrm{M}}=E_{\mathrm{e}}^{\mathrm{M}}+E_{\mathrm{ZPVE}}^{\mathrm{M}}
$$

where $E_{\mathrm{e}}^{\mathrm{M}}$ is the total NR electronic energy of $\mathrm{M}$ if the nuclei were held fixed at positions corresponding to the potential minimum and $E_{\mathrm{ZPVE}}^{\mathrm{M}}$ is the zero-point vibrational energy. $E_{\mathrm{e}}^{\mathrm{M}}$ can be written as

$$
E_{\mathrm{e}}^{\mathrm{M}}=E_{\mathrm{HF}}^{\mathrm{M}}+E_{\mathrm{corr}}^{\mathrm{M}}
$$

where $E_{\mathrm{HF}}^{\mathrm{M}}$ is the $\mathrm{HF}$ energy and $E_{\mathrm{corr}}^{\mathrm{M}}$ the correlation energy of $\mathrm{M}$. Given the atomization energies, the relativistic corrections, the zero-point corrections, the total atomic energies and the HF energies, we can now determine $E_{0}^{\mathrm{M}}, E_{\mathrm{e}}^{\mathrm{M}}$ and $E_{\text {corr }}^{\mathrm{M}}$,

$$
\begin{aligned}
E_{0}^{\mathrm{M}} & =\sum_{i}^{N} E_{\mathrm{e}}^{i}+\Delta E_{\mathrm{Rel}}-\Sigma D_{0}, \\
E_{\mathrm{e}}^{\mathrm{M}} & =E_{0}^{\mathrm{M}}-E_{\mathrm{ZPVE}}^{\mathrm{M}}, \\
E_{\mathrm{corr}}^{\mathrm{M}} & =E_{\mathrm{e}}^{\mathrm{M}}-E_{\mathrm{HF}}^{\mathrm{M}} .
\end{aligned}
$$


Table 1. Methods used to derive required quantities.

\begin{tabular}{ll}
\hline Quantity & \multicolumn{1}{c}{ Method } \\
\hline$\Sigma D_{0}$ & Experimental \\
$E_{\mathrm{ZPVE}}$ & MP2/6-31G* ZPVE scaled by 0.9661 \\
$E_{\mathrm{HF}}$ & HF/cc-pV5Z-h//(Exp. or QCISD $/$ \\
& G3MP2Large) \\
$\Delta E_{\mathrm{Rel}}$ & CISD $(\mathrm{FC}) /$ cc-pVTZ//CCSD $(\mathrm{T})(\mathrm{FC}) /$ \\
& aug-cc-pVTZ for one-electron scalar \\
& relativistic corrections. \\
& Spin-orbit corrections from experiment \\
$E_{\mathrm{e}}$ & Total atomic energies from [18] \\
\hline
\end{tabular}

\section{Results}

The quality of the results we obtain using equations (4)-(6) is clearly dependent on the accuracy of the data we use. The methods used to obtain these data are summarized in table 1 . We obtain the atomization energy from experiment, specifically we take those listed in [4] for the G1 molecules (correcting the value for $\mathrm{CN}$ to that quoted in [12]) and the value for $\mathrm{H}_{2}$ from [13]. The majority of these have error bars less than $1 \mathrm{~m} E_{\mathrm{h}}$ and we assume they are accurate for our purposes.

Table 2. Geometry ${ }^{\mathrm{a}}$, atomization energy $\Sigma D_{0}{ }^{\mathrm{b}}$, zero-point vibrational energy $E_{\mathrm{ZPVE}}{ }^{\mathrm{c}}$, relativistic correction $\Delta E_{\mathrm{Rel}}{ }^{\mathrm{d}}$, non-relativistic energy of lowest vibronic state $E_{0}$, non-relativistic electronic energy $E_{\mathrm{e}}^{\mathrm{e}}, \mathrm{HF}$ energy $E_{\mathrm{HF}}{ }^{\mathrm{f}}$, and correlation energy $E_{\text {corr }}$ for atoms and molecules. All energies are in Hartrees.

\begin{tabular}{|c|c|c|c|c|c|c|c|c|c|c|}
\hline \multirow[t]{2}{*}{ Species } & \multirow[t]{2}{*}{ Geom. } & \multirow[t]{2}{*}{$\Sigma D_{0} \times 10^{3}$} & \multirow[t]{2}{*}{$E_{\mathrm{ZPVE}} \times 10^{3}$} & \multirow[t]{2}{*}{$\Delta E_{\mathrm{Rel}} \times 10^{3}$} & \multirow[t]{2}{*}{$E_{0}$} & \multirow[t]{2}{*}{$E_{\mathrm{e}}$} & \multicolumn{2}{|c|}{$E_{\mathrm{HF}}$} & \multicolumn{2}{|c|}{$E_{\text {corr }} \times 10^{3}$} \\
\hline & & & & & & & $\mathrm{UHF}$ & RHF & $\mathrm{UHF}$ & RHF \\
\hline $\mathrm{H}$ & - & - & - & - & - & -0.5000 & -0.5000 & -0.5000 & -0 & -0 \\
\hline $\mathrm{He}$ & - & - & - & - & - & -2.9037 & -2.8616 & -2.8616 & -42 & -42 \\
\hline $\mathrm{Li}$ & - & - & - & - & - & -7.4781 & -7.4327 & -7.4327 & -45 & -45 \\
\hline $\mathrm{Be}$ & - & - & - & - & - & -14.6674 & -14.5730 & -14.5730 & -94 & -94 \\
\hline B & - & - & - & - & - & -24.6539 & -24.5331 & -24.5291 & -121 & -125 \\
\hline $\mathrm{C}$ & - & - & - & - & - & -37.8450 & -37.6937 & -37.6886 & -151 & -156 \\
\hline $\mathrm{N}$ & - & - & - & - & - & -54.5892 & -54.4045 & -54.4009 & -185 & -188 \\
\hline $\mathrm{O}$ & - & - & - & - & - & -75.0673 & -74.8188 & -74.8122 & -249 & -255 \\
\hline $\mathrm{F}$ & - & - & - & - & - & -99.7339 & -99.4161 & -99.4112 & -318 & -323 \\
\hline $\mathrm{Ne}$ & - & - & - & - & - & -128.9376 & -128.5468 & -128.5468 & -391 & -391 \\
\hline $\mathrm{Na}$ & - & - & - & - & - & -162.2546 & -161.8587 & -161.8587 & -396 & -396 \\
\hline $\mathrm{Mg}$ & - & - & - & - & - & -200.0530 & -199.6146 & -199.6146 & -438 & -438 \\
\hline $\mathrm{Al}$ & - & - & - & - & - & -242.3460 & -241.8808 & -241.8768 & -465 & -469 \\
\hline $\mathrm{Si}$ & - & - & - & - & - & -289.3590 & -288.8588 & -288.8544 & -500 & -505 \\
\hline $\mathrm{P}$ & - & - & - & - & - & -341.2590 & -340.7192 & -340.7187 & -540 & -540 \\
\hline $\mathrm{S}$ & - & - & - & - & - & -398.1100 & -397.5132 & -397.5071 & -597 & -603 \\
\hline $\mathrm{Cl}$ & - & - & - & - & - & -460.1480 & -459.4897 & -459.4838 & -658 & -664 \\
\hline $\mathrm{Ar}$ & - & - & - & - & - & -527.5400 & -526.8173 & -526.8173 & -723 & -723 \\
\hline $\mathrm{H}_{2}$ & $\mathrm{HH}$ & 164.6 & 9.8 & 0.0 & -1.1646 & -1.1745 & -1.1336 & -1.1336 & -41 & -41 \\
\hline $\mathrm{LiH}$ & $\mathrm{HH}$ & 89.2 & 3.1 & 0.0 & -8.0673 & -8.0704 & -7.9873 & -7.9873 & -83 & -83 \\
\hline $\mathrm{BeH}$ & $\mathrm{HH}$ & 74.7 & 4.7 & 0.0 & -15.2421 & -15.2468 & -15.1536 & -15.1532 & -93 & -94 \\
\hline $\mathrm{CH}$ & $\mathrm{HH}$ & 127.3 & 6.5 & 0.0 & -38.4723 & -38.4788 & -38.2844 & -38.2798 & -194 & -199 \\
\hline $\mathrm{CH}_{2}\left({ }^{3} \mathrm{~B}_{1}\right)$ & $\mathrm{KU}$ & 286.2 & 16.7 & -0.5 & -39.1317 & -39.1484 & -38.9408 & -38.9353 & -208 & -213 \\
\hline $\mathrm{CH}_{2}\left({ }^{1} \mathrm{~A}_{1}\right)$ & $\mathrm{KU}$ & 271.9 & 17.4 & -0.3 & -39.1172 & -39.1346 & -38.8959 & -38.8959 & -239 & -239 \\
\hline $\mathrm{CH}_{3}$ & $\mathrm{KU}$ & 460.9 & 29.5 & -0.2 & -39.8060 & -39.8355 & -39.5811 & -39.5766 & -254 & -259 \\
\hline $\mathrm{CH}_{4}$ & KU & 625.5 & 44.8 & -0.5 & -40.4710 & -40.5158 & -40.2170 & -40.2170 & -299 & -299 \\
\hline $\mathrm{NH}$ & $\mathrm{HH}$ & 125.9 & 7.4 & -0.2 & -55.2153 & -55.2227 & -54.9862 & -54.9783 & -236 & -244 \\
\hline $\mathrm{NH}_{2}$ & KU & 270.9 & 18.9 & -0.3 & -55.8604 & -55.8794 & -55.5920 & -55.5870 & -287 & -292 \\
\hline $\mathrm{NH}_{3}$ & QCI & 440.9 & 34.1 & -0.5 & -56.5306 & -56.5647 & -56.2249 & -56.2249 & -340 & -340 \\
\hline $\mathrm{OH}$ & $\mathrm{HH}$ & 161.4 & 8.2 & -0.2 & -75.7289 & -75.7371 & -75.4278 & -75.4228 & -309 & -314 \\
\hline $\mathrm{OH}_{2}$ & KU & 349.5 & 20.8 & -0.8 & -76.4176 & -76.4383 & -76.0672 & -76.0672 & -371 & -371 \\
\hline $\mathrm{FH}$ & $\mathrm{HH}$ & 215.5 & 8.9 & -1.0 & -100.4503 & -100.4592 & -100.0706 & -100.0706 & -389 & -389 \\
\hline $\mathrm{SiH}_{2}\left({ }^{1} \mathrm{~A}_{1}\right)$ & QCI & 230.1 & 11.8 & -1.0 & -290.5901 & -290.6019 & -290.0352 & -290.0352 & -567 & -567 \\
\hline $\mathrm{SiH}_{2}\left({ }^{3} \mathrm{~B}_{1}\right)$ & KU & 196.7 & 12.2 & -1.3 & -290.5569 & -290.5692 & -290.0288 & -290.0268 & -540 & -542 \\
\hline $\mathrm{SiH}_{3}$ & QCI & 341.0 & 20.4 & -1.3 & -291.2013 & -291.2217 & -290.6467 & -290.6451 & -575 & -577 \\
\hline $\mathrm{SiH}_{4}$ & QCI & 482.5 & 31.3 & -1.6 & -291.8431 & -291.8744 & -291.2682 & -291.2682 & -606 & -606 \\
\hline $\mathrm{PH}_{2}$ & QCI & 230.6 & 13.6 & -0.3 & -342.4899 & -342.5035 & -341.8929 & -341.8872 & -611 & -616 \\
\hline $\mathrm{PH}_{3}$ & $\mathrm{KU}$ & 362.4 & 24.2 & -0.6 & -343.1220 & -343.1462 & -342.4943 & -342.4943 & -652 & -652 \\
\hline $\mathrm{SH}_{2}$ & KU & 276.0 & 15.2 & -1.4 & -399.3874 & -399.4026 & -398.7198 & -398.7198 & -683 & -683 \\
\hline $\mathrm{ClH}$ & $\mathrm{HH}$ & 162.9 & 6.7 & -1.6 & -460.8125 & -460.8192 & -460.1125 & -460.1125 & -707 & -707 \\
\hline $\mathrm{Li}_{2}$ & $\mathrm{HH}$ & 38.2 & 0.8 & 0.0 & -14.9944 & -14.9951 & -14.8716 & -14.8716 & -124 & -124 \\
\hline
\end{tabular}


Table 2. Continued.

\begin{tabular}{|c|c|c|c|c|c|c|c|c|c|c|}
\hline \multirow[t]{2}{*}{ Species } & \multirow[t]{2}{*}{ Geom. } & \multirow[t]{2}{*}{$\Sigma D_{0} \times 10^{3}$} & \multirow[t]{2}{*}{$E_{\mathrm{ZPVE}} \times 10^{3}$} & \multirow[t]{2}{*}{$\Delta E_{\mathrm{Rel}} \times 10^{3}$} & \multirow[t]{2}{*}{$E_{0}$} & \multirow[t]{2}{*}{$E_{\mathrm{e}}$} & \multicolumn{2}{|c|}{$E_{\mathrm{HF}}$} & \multicolumn{2}{|c|}{$E_{\text {corr }} \times 10^{3}$} \\
\hline & & & & & & & UHF & RHF & UHF & RHF \\
\hline $\mathrm{LiF}$ & $\mathrm{HH}$ & 219.3 & 2.2 & -1.0 & -107.4322 & -107.4344 & -106.9931 & -106.9931 & -441 & -441 \\
\hline $\mathrm{HCCH}$ & QCI & 619.8 & 25.0 & -0.8 & -77.3105 & -77.3355 & -76.8555 & -76.8555 & -480 & -480 \\
\hline $\mathrm{H}_{2} \mathrm{CCH}_{2}$ & QCI & 847.6 & 50.3 & -0.8 & -78.5384 & -78.5888 & -78.0706 & -78.0706 & -518 & -518 \\
\hline $\mathrm{H}_{3} \mathrm{CCH}_{3}$ & QCI & 1061.8 & 74.6 & -1.0 & -79.7528 & -79.8274 & -79.2665 & -79.2665 & -561 & -561 \\
\hline $\mathrm{CN}$ & $\mathrm{HH}$ & 284.3 & 6.3 & -0.2 & -92.7187 & -92.7250 & -92.2422 & -92.2250 & -483 & -500 \\
\hline $\mathrm{HCN}$ & KU & 480.9 & 15.5 & -0.5 & -93.4156 & -93.4311 & -92.9157 & -92.9157 & -515 & -515 \\
\hline $\mathrm{CO}$ & $\mathrm{HH}$ & 408.3 & 4.7 & -0.8 & -113.3214 & -113.3261 & -112.7907 & -112.7907 & -535 & -535 \\
\hline $\mathrm{HCO}$ & $\mathrm{KU}$ & 430.8 & 13.0 & -1.0 & -113.8440 & -113.8570 & -113.3035 & -113.2981 & -553 & -559 \\
\hline $\mathrm{H}_{2} \mathrm{CO}$ & QCI & 569.2 & 26.4 & -1.1 & -114.4826 & -114.5090 & -113.9231 & -113.9231 & -586 & -586 \\
\hline $\mathrm{H}_{3} \mathrm{COH}$ & QCI & 766.2 & 50.8 & -1.3 & -115.6798 & -115.7306 & -115.1017 & -115.1017 & -629 & -629 \\
\hline $\mathrm{N}_{2}$ & $\mathrm{HH}$ & 358.7 & 4.8 & -0.2 & -109.5373 & -109.5421 & -108.9929 & -108.9929 & -549 & -549 \\
\hline $\mathrm{H}_{2} \mathrm{NNH}_{2}$ & QCI & 646.0 & 52.9 & -0.3 & -111.8248 & -111.8776 & -111.2364 & -111.2364 & -641 & -641 \\
\hline $\mathrm{NO}$ & $\mathrm{HH}$ & 239.2 & 8.6 & -0.5 & -129.8962 & -129.9047 & -129.3087 & -129.3009 & -596 & -604 \\
\hline $\mathrm{O}_{2}$ & $\mathrm{HH}$ & 188.0 & 3.1 & -1.0 & -150.3236 & -150.3267 & -149.6908 & -149.6672 & -636 & -660 \\
\hline $\mathrm{HOOH}$ & QCI & 402.1 & 25.4 & -1.4 & -151.5381 & -151.5635 & -150.8528 & -150.8528 & -711 & -711 \\
\hline $\mathrm{F}_{2}$ & $\mathrm{HH}$ & 58.8 & 2.2 & -1.4 & -199.5280 & -199.5303 & -198.7729 & -198.7729 & -757 & -757 \\
\hline $\mathrm{CO}_{2}$ & $\mathrm{KU}$ & 608.6 & 11.2 & -1.8 & -188.5899 & -188.6011 & -187.7250 & -187.7250 & -876 & -876 \\
\hline $\mathrm{Na}_{2}$ & $\mathrm{HH}$ & 26.5 & 0.4 & 0.0 & -324.5357 & -324.5360 & -323.7165 & -323.7165 & -819 & -819 \\
\hline $\mathrm{Si}_{2}$ & $\mathrm{HH}$ & 117.9 & 1.1 & -1.6 & -578.8375 & -578.8386 & -577.7615 & -577.7549 & -1077 & -1084 \\
\hline $\mathrm{P}_{2}$ & $\mathrm{HH}$ & 185.0 & 1.6 & 0.3 & -682.7027 & -682.7043 & -681.4995 & -681.4995 & -1205 & -1205 \\
\hline $\mathrm{S}_{2}$ & $\mathrm{HH}$ & 160.5 & 1.5 & -2.2 & -796.3827 & -796.3842 & -795.1090 & -795.0933 & -1275 & -1291 \\
\hline $\mathrm{Cl}_{2}$ & $\mathrm{HH}$ & 91.2 & 1.2 & -1.6 & -920.3887 & -920.3899 & -919.0101 & -919.0101 & -1380 & -1380 \\
\hline $\mathrm{NaCl}$ & $\mathrm{HH}$ & 155.4 & 0.8 & -1.8 & -622.5597 & -622.5605 & -621.4600 & -621.4600 & -1101 & -1101 \\
\hline $\mathrm{SiO}$ & $\mathrm{HH}$ & 303.6 & 2.6 & -1.3 & -364.7312 & -364.7337 & -363.8545 & -363.8545 & -879 & -879 \\
\hline $\mathrm{SC}$ & $\mathrm{HH}$ & 270.1 & 2.9 & -1.1 & -436.2262 & -436.2291 & -435.3618 & -435.3618 & -867 & -867 \\
\hline $\mathrm{SO}$ & $\mathrm{HH}$ & 196.8 & 2.4 & -1.8 & -473.3759 & -473.3783 & -472.4210 & -472.4039 & -957 & -974 \\
\hline $\mathrm{ClO}$ & $\mathrm{HH}$ & 100.9 & 1.9 & -2.1 & -535.3182 & -535.3201 & -534.3183 & -534.3106 & -1002 & -1009 \\
\hline $\mathrm{ClF}$ & $\mathrm{HH}$ & 96.1 & 1.8 & -2.2 & -559.9802 & -559.9820 & -558.9194 & -558.9194 & -1063 & -1063 \\
\hline $\mathrm{Si}_{2} \mathrm{H}_{6}$ & QCI & 797.0 & 49.1 & -3.2 & -582.5181 & -582.5672 & -581.3842 & -581.3842 & -1183 & -1183 \\
\hline $\mathrm{CH}_{3} \mathrm{Cl}$ & KU & 591.2 & 37.8 & -2.2 & -500.0865 & -500.1243 & -499.1564 & -499.1564 & -968 & -968 \\
\hline $\mathrm{CH}_{3} \mathrm{SH}$ & QCI & 709.3 & 46.2 & -1.9 & -438.6662 & -438.7124 & -437.7665 & -437.7665 & -946 & -946 \\
\hline $\mathrm{HOCl}$ & KU & 249.1 & 12.7 & -2.4 & -535.9668 & -535.9795 & -534.9340 & -534.9340 & -1045 & -1045 \\
\hline $\mathrm{SO}_{2}$ & KU & 404.8 & 6.3 & -3.0 & -548.6524 & -548.6588 & -547.3250 & -547.3250 & -1334 & -1334 \\
\hline
\end{tabular}

${ }^{\mathrm{a}}$ Geometries are denoted by $\mathrm{HH}=$ Huber and Herzberg (experimental $r_{\mathrm{e}}$ from [13]), KU = Kuchitsu (experimental $r_{\mathrm{e}}$ from [19]) and QCI = geometry calculated using QCISD/G3MP2Large.

${ }^{\mathrm{b}}$ Taken from [4], with the exception of $\mathrm{CN}$ from [12] and $\mathrm{H}_{2}$ from [13].

${ }^{\mathrm{c}} \mathrm{MP} 2 / 6-31 \mathrm{G}^{*}$ ZPVE scaled by 0.9661 [14] taken from [15].

${ }^{\mathrm{d}}$ Scalar relativistic and spin-orbit corrections taken from [16].

eAtomic total energies taken from [18].

${ }^{\mathrm{f}}$ Calculated using cc-pV5Z- $h$.

The zero-point vibrational energies are obtained by scaling the ZPVE from a MP2/6-31G* harmonic frequency calculation by 0.9661 [14] and are taken from [15]. The RMS error of this method with the 39 molecules used to parameterize it is approximately $0.3 \mathrm{~m} E_{\mathrm{h}}$.

We use the relativistic corrections to the atomization energies listed by Feller and Peterson [16] which consist of scalar relativistic corrections (one-electron Darwin and mass-velocity terms in the Breit-Pauli Hamiltonian) obtained using a CISD(FC)/cc-pVTZ// $\operatorname{CCSD}(\mathrm{T})(\mathrm{FC}) /$ aug-cc-pVTZ wavefunction, and also a spin-orbit correction based on experimental results. The scalar relativistic corrections are expected to be within
$1 \mathrm{~m} E_{\mathrm{h}}$ of four-component or Douglas-Kroll results. Although the geometries at which the relativistic corrections are calculated are not the experimental geometries, the differences are expected to be negligible and, furthermore, it has been shown that these corrections have a very weak dependence on geometry [17].

The NR electronic energies of the atoms are taken from [18] and are taken to have errors of less than $1 \mathrm{~m} E_{\mathrm{h}}$. The molecular HF energies are calculated using experimental $r_{\mathrm{e}}$ structures (taken from $[13,19]$ ) which are available for 42 of the species under investigation. The remaining $14 \mathrm{HF}$ energies are calculated at the QCISD/G3MP2Large [20-22] geometries. The HF results are obtained using the cc-pV5Z [23, 24] basis 
set with the $h$-functions removed (cc-pV5Z- $h$ ). Halkier et al. have studied the convergence of the HF energy using correlation consistent basis sets and report that using the cc-pV5Z basis yields energies within $1 \mathrm{~m} E_{\mathrm{h}}$ of the HF limit [25]. All HF energies are calculated using the Q-CHEM package [26].

In table 2 we present all of the quantities described above. The correlation energy depends upon whether a restricted (RHF) or unrestricted (UHF) wavefunction is used and we therefore list both. We note that the atomic correlation energies given here are different to those given by Chakravorty et al. [18] as they have used numerical HF energies [27] which are symmetry restricted and therefore higher than either the RHF or UHF energies shown here. We would recommend, when assessing a post-HF method using a RHF/UHF wavefunction to estimate the correlation energy, the use of the energies listed here rather than those of [18].

Ideally, we would like to be able to compare our results with exact solutions (within an infinite basis set) of the Schrödinger equation, but in the two decades since this was done for water [28] (within a double-zeta basis set) we have not moved much closer to this goal. Although quantum Monte Carlo offers estimates to the exact energy, quantifying the error due to the fixed node approximation requires the use of experimentally derived energies like the ones listed in the table. We hope that the data listed here will not only be useful, but also highlight the need for theoretical methods which can produce benchmark data like these without approximation or experimental data.

\section{Acknowledgements}

This research was partly supported by the Engineering and Physical Sciences Research Council through a project studentship (GR/R81121) to D.P.O. We would also like to thank the EPSRC for JREI funding for computer resources.

\section{References}

[1] P.J. Stephens, F.J. Devlin, C.F. Chablowski, M.J. Frisch, J. phys. Chem., 98, 11623 (1994).

[2] J.A. Pople, M. Head-Gordon, D.J. Fox, K. Raghavachari, L.A. Curtiss, J. chem. Phys., 90, 5622 (1989).
[3] L.A. Curtiss, C. Jones, G.W. Trucks, K. Raghavachari, J.A. Pople, J. chem. Phys., 93, 2537 (1990).

[4] L.A. Curtiss, K. Raghavachari, G.W. Trucks, J.A. Pople, J. chem. Phys., 94, 7221 (1991).

[5] L.A. Curtiss, K. Raghavachari, P.C. Redfern, V. Rassolov, J.A. Pople, J. chem. Phys., 109, 7764 (1998).

[6] P.J. Reynolds, D.M. Ceperley, B.J. Alder, W.A. Lester, J. chem. Phys., 77, 5593 (1982).

[7] P.J. Reynolds, M. Dupuis, W.A. Lester, J. chem. Phys., 82, 1983 (1985).

[8] D. Feller, C.M. Boyle, E.R. Davidson, J. chem. Phys., 86, 3424 (1986).

[9] C. Filippi, C.J. Umrigar, J. chem. Phys., 105, 213 (1996).

[10] A. Lüchow, J.B. Anderson, D. Feller, J. chem. Phys., 106, 7706 (1997).

[11] S.-I. Lu, J. chem. Phys., 21, 9528 (2003).

[12] Y. Huang, S.A. Barts, J.B. Halpern, J. phys. Chem., 96, 425 (1992).

[13] K.P. Huber, G. Herzberg, Molecular Spectra and Molecular Structure: Constants of Diatomic Molecules, Vol. 4, Van Nostrand Reinhold, New York (1979).

[14] A.P. Scott, L. Radom, J. phys. Chem., 100, 16502 (1996).

[15] L.A. Curtiss, K. Raghavachari, J.A. Pople, J. chem. Phys., 103, 4192 (1995).

[16] D. Feller, K.A. Peterson, J. chem. Phys., 110, 8384 (1999).

[17] J.M.L. Martin, A. Sundermann, P.L. Fast, D.G. Truhlar, J. chem. Phys., 113, 1348 (2000).

[18] S.J. Chakravorty, S.R. Gwaltney, E.R. Davidson, F.A. Parpia, C. Froese-Fischer, Phys. Rev. A, 47, 3649 (1993).

[19] K. Kuchitsu (Ed.), Structure of Free Polyatomic Molecules: Basic Data, Springer, Berlin (1998).

[20] J.A. Pople, M. Head-Gordon, K. Raghavachari, J. chem. Phys., 87, 5968 (1987).

[21] L.A. Curtiss, P.C. Redfern, K. Raghavachari, V. Rassolov, J.A. Pople, J. chem. Phys., 110, 4703 (1999).

[22] http://comp.chem.umn.edu/database.

[23] T.H. Dunning Jr, J. chem. Phys., 90, 1007 (1989).

[24] D.E. Woon, T.H. Dunning Jr, J. chem. Phys., 100, 2975 (1994).

[25] A. Halkier, T. Helgaker, P. Jørgensen, W. Klopper, J. Olsen, Chem. Phys. Lett., 302, 437 (1999).

[26] J. Kong, C.A. White, A.I. Krylov, C.D. Sherrill, R.D. Adamson, T.R. Furlani, M.S. Lee, A.M. Lee, S.R. Gwaltney, T.R. Adams, C. Ochsenfeld, A.T.B. Gilbert, G.S. Kedziora, V.A. Rassolov, D.R. Maurice, N. Nair, Y. Shao, N.A. Besley, P.E. Maslen, J.P. Dombroski, H. Daschel, W. Zhang, P.P. Korambath, J. Baker, E.F.C. Byrd, T. van Voorhis, M. Oumi, S. Hirata, C.-P. Hsu, N. Ishikawa, J. Florian, A. Warshel, B.G. Johnson, P.M.W. Gill, M. HeadGordon, J.A. Pople, J. comput. Chem., 21, 1532 (2000).

[27] C. Froese-Fischer, The Hartree-Fock Method for Atoms: A Numerical Approach, Wiley, New York (1977).

[28] P. Saxe, H.F. Schaefer, N.C. Handy, Chem. Phys. Lett., 79, 202 (1981). 